\title{
Existence conditions for symmetric strong vector quasi-equilibrium problems
}

\author{
Le Xuan Dai ${ }^{1}{ }^{*}$, Nguyen Van Hung ${ }^{2}$
}

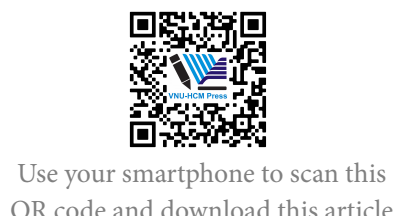

QR code and download this article

${ }^{1}$ Department of Applied Mathematics,

Ho Chi Minh City University of

Technology, VNUHCM, Vietnam

${ }^{2}$ Department of Scientific Fundamentals, Posts and Telecommunications Institute of Technology, Ho Chi Minh City, Vietnam

Correspondence

Le Xuan Dai, Department of Applied Mathematics, Ho Chi Minh City University of Technology, VNUHCM, Vietnam

Email: ytkadai@hcmut.edu.vn

History

- Received: 6-12-2019

- Accepted: 18-12-2020

- Published: 31-12-2020

DOI :10.32508/stdjet.v3iSI3.638

\section{Check for updates}

\section{Copyright}

(c) VNU-HCM Press. This is an openaccess article distributed under the terms of the Creative Commons Attribution 4.0 International license.

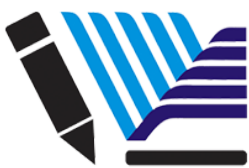

VNU-HCM Press

\begin{abstract}
In the following paper, the symmetric strong vector quasi-equilibrium problems will be studied thoroughly. Afterward, the existence conditions of solution sets for these problems has been established. The results which are presented in this paper improve and extend the main results mentioned in the literature. Our results can be illustrated by some interesting examples.

In 1994, Noor and Oettli introduced the following the symmetric scalar quasi-equilibrium problem. This problem is one of the generalization of the symmetric scalar quasi-equilibrium problem which is presented by Noor and Oettli. Since then, the symmetric vector quasi-equilibrium problem has been investigated by a huge number of authors in different ways.

The research works mentioned above are one of our motivation to improve and extend the problem. So, in this paper, we will introduce the vector quasi-equilibrium problems. Afterward, some existence conditions of solution sets for these problems will be established. The symmetric vector quasi-equilibrium problems consist of many optimization - related models namely symmetric vector quasi-variational inequality problems, fixed point problems, coincidence-point problems and complementarity problems, etc. In recent years, a lot of results for existence of solutions for symmetric vector quasi-equilibrium problems, vector quasi-equilibrium problems, vector quasivariational inequality problems and optimization problems have been established by many authors in different ways.

We will present our work in the following steps. In the first section of our paper, we will introduce the model of symmetric vector quasi-equilibrium problems. In the following section, we recall definitions, lemmas which can be used for the main results. In the last section, we will establish some conditions for existence and closedness of the solutions set by applying fixed-point theorem for symmetric vector quasi-equilibrium problems.

The results presented in this paper improve and extend the main results in the literature. Some examples are given to illustrate our results. Hence our results, Theorem 3.1 and Theorem 3.6 have significant improvements.
\end{abstract}

Key words: Symmetric generalized quasi-equilibrium problems, Kakutani-Fan-Glicksberg fixedpoint theorem, Closedness

\section{INTRODUCTION}

In 1994, Noor and Oettli ${ }^{1}$ presented the following the symmetric scalar quasi-equilibrium problem which consists of finding $(\bar{x}, \bar{y}) \in A \times B$ such that $\bar{x} \in$ $S(\bar{x}, \bar{y}), \bar{y} \in T(\bar{x}, \bar{y})$

and

$f(x, \bar{y}) \geq f(\bar{x}, \bar{y})$, for all $x \in S(\bar{x}, \bar{y})$,

$g(\bar{x}, y) \geq g(\bar{x}, \bar{y})$, for all $y \in T(\bar{x}, \bar{y})$,

where $X, Y$ are real locally convex Hausdorff topological vector spaces and $A \subset X, B \subset Y$ are non-empty sets, $S: A \times B \rightrightarrows A, T: A \times B \rightrightarrows B$ are set-valued mappings and $f, g: A \times B \rightarrow R$ are real functions.

In 2003, the symmetric vector quasi-equilibrium problem (in short, (SVQEP)) has been introduced and investigated by $\mathrm{Fu}^{2}$. Let $X, Y$ and $Z$ be real locally convex Hausdorff topological vector spaces, and let $A \subset X$,
$B \subset Y$ be non-empty sets and $C \subset Z$ be a closed convex point cone with int $C \neq \varnothing$, where int $C$ denotes the interior of $C$.

Let $S: A \times B \rightrightarrows A, T: A \times B \rightrightarrows B$ be set-valued mappings and $\mathrm{f}, \mathrm{g}: A \times B \longrightarrow Z$ be vector functions. Find $(\overline{\mathrm{x}}, \overline{\mathrm{y}}) \in A \times B$ such that $\bar{x} \in S(\bar{x}, \bar{y}), \bar{y} \in T(\bar{x}, \bar{y})$ and $f(x, \bar{y})-f(\bar{x}, \bar{y}) \notin-\operatorname{int} C$, for all $x \in S(\bar{x}, \bar{y})$, $g(\bar{x}, y)-g(\bar{x}, \bar{y}) \notin-\operatorname{int} C$, for all $y \in T(\bar{x}, \bar{y})$.

The problem is one of the generalization of the symmetric scalar quasi-equilibrium problem by Noor and Oettli ${ }^{1}$. Since then, many authors have studied the symmetric vector quasi-equilibrium problem in different ways, see ${ }^{3,4}$ and the references therein.

In this paper, we will introduce the vector quasiequilibrium problems. Afterward, we will point out some existence conditions of solution sets for these problems. Now, we move on to our problem setting. 
Let $X, Y, Z$ be real locally convex Hausdorff topological vector spaces, $A \subset X, B \subset Y$ be non-empty compact subsets and $C \subset Z$ be a closed convex point cone with int $C \neq \varnothing$, where int $C$ denotes the interior of $C$. Let $K: A \times B \rightrightarrows A, T: A \times B \rightrightarrows B$ be multi-functions and $f: A \times B \times A \rightarrow Z, g: B \times A \times B \rightarrow Z$

be vector functions. We will study the following symmetric vector quasi-equilibrium problems (in short, (SQVEP).

(SQVEP): Find $(\bar{x}, \bar{y}) \in A \times B$ such that $\bar{x} \in$ $S(\bar{x}, \bar{y}), \bar{y} \in T(\bar{x}, \bar{y})$ and

$$
\begin{aligned}
& f\left(\bar{x}, \bar{y}, x^{*}\right) \in C, \forall x^{*} \in K(\bar{x}, \bar{y}), \\
& g\left(\bar{y}, \bar{x}, y^{*}\right) \in C, \forall y^{*} \in T(\bar{x}, \bar{y}),
\end{aligned}
$$

We denote that $\Omega$ is the solution set of (SQVEP). Note that the symmetric vector quasi-equilibrium problems include many optimization - related models, for instance, symmetric vector quasi-variational inequality problems, vector quasi-equilibrium problems, variational inequality problems, Nash equilibria problems, fixed point problems, coincidence-point problems and complementarity problems, etc. In recent years, a lot of results for existence of solutions for symmetric vector quasi-equilibrium problems, vector quasi-equilibrium problems, vector quasi-variational inequality problems and optimization problems have been established by many authors in different ways. For more details on this topic, we refer the readers to ${ }^{3-5}$ and the references therein.

Our paper is presented in the following way. Firstly, we introduce the model symmetric vector quasiequilibrium problems. Secondly, we recall definitions, lemmas which can be used to proof the main theorem. Lastly, we establish some existence and closedness theorems by using fixed-point theorem for symmetric vector quasi-equilibrium problems.

\section{PRELIMINARIES}

In this section, we recall some basic definitions and some of their properties.

Definition $2.1^{6}$ Let $X, Y$ be two topological vector spaces, $A$ be a nonempty subset of $X$ and $F: A \rightrightarrows Y$ be a multi-function.

a) $F$ is said to be lower semi-continuous (lsc) at $x_{0} \in A$ if $F\left(x_{0}\right) \cap U \neq \varnothing$ for some open set $U \subset Y$ implies the existence of a neighborhood $N$ of $x_{0}$ such that $F(x) \cap$ $U \neq \varnothing, \forall x \in N$.

b) $F$ is said to be lower semi-continuous in $A$ if it is lower semicontinuous at all $x_{0} \in A$.

c) $F$ is said to be upper semi-continuous (usc) at $x_{0} \in A$ if for each open set $U \supset F\left(x_{0}\right)$, there is a neighborhood $N$ of $x_{0}$ such that

$$
U \supset F(x), \forall x \in N
$$

d) $F$ is said to be upper semi-continuous in $S$ if it is upper semicontinuous at all $x_{0} \in A$.

e) $F$ is said to be continuous in $A$ if it is both lsc and usc in $A$.

f) $F$ is said to be closed if

$$
\operatorname{Graph}(F)=\{(x, y): x \in A, y \in F(x)\}
$$

is a closed subset in $A \times Y$.

Lemma $2.1^{7}$ Let $X, Y$ be two topological vector spaces, $A$ be a nonempty convex subset of $X$ and $F: A \rightrightarrows Y$ be a multifunction.

a) If $F$ is upper semicontinuous at $x_{0} \in A$ with closed values, then $F$ is closed at $x_{0} \in A$.

b) If $F$ is closed at $x_{0} \in A$ and $Y$ is compact, then $F$ is upper semi-continuous at $x_{0} \in A$.

c) If $F$ has compact values, then $F$ is usc at $x_{0} \in A$ if and only if, for each net $\left\{x_{\alpha}\right\} \subseteq A$ which converges to $x_{0} \in$ $A$ and for each net $\left\{y_{\alpha}\right\} \subseteq F\left(x_{\alpha}\right)$, there are $y_{0} \in F\left(x_{0}\right)$ and a subnet $\left\{y_{\beta}\right\}$ of $\left\{y_{\alpha}\right\}$ such that $y_{\beta} \longrightarrow y_{0}$.

Definition $2.2^{8}$ Let $X$ and $Z$ be two topological vector spaces and $A \subseteq X$ be non-empty convex set, $C \subset Z$ is a nonempty closed convex cone. Suppose $f: A \longrightarrow Z$ be a vector function. $f$ is called properly $\mathrm{C}$-quasi-convex in $A$ if and only if, for every $\mathrm{x}_{1}, x_{2} \in A$ and each $\lambda \in$ $[0,1]$, we have

$$
\begin{aligned}
& \text { either } f\left(\lambda x_{1}+(1-\lambda) x_{2}\right) \leq f\left(x_{1}\right), \\
& \text { or } \quad f\left(\lambda x_{1}+(1-\lambda) x_{2}\right) \leq f\left(x_{2}\right) .
\end{aligned}
$$

Definition $2.3^{7}$ Let $X$ and $Z$ be two topological vector spaces and $A \subseteq X$ be nonempty convex set, $C \subset Z$ is a non-empty closed convex cone. $A$ mapping $f: A \longrightarrow Z$ is called $C$-continuous at $x_{0} \in A$ if, for any open neighborhood $V$ of 0 in $Z$, there exists an open neighborhood $\mathrm{U}$ of $x_{0}$ in $A$ such that

$$
f(x) \in f\left(x_{0}\right)+V+C, \forall x \in U \cap A,
$$

and C-continuous in $\mathrm{A}$ if it is $\mathrm{C}$-continuous at every point of A.

Lemma $2.2^{9}$ Let $A$ be a nonempty convex compact subset of Hausdorff topological vector space $X$ and $M$ be a subset of $A \times A$ such that

a) for each at $x \in A,(x, x) \notin M$;

b) for each at $y \in A$, the set $\{x \in A:(x, x) \in M\}$ is open in $A$;

c) for each at $x \in A$, the set $\{y \in A:(x, x) \in M\}$ is convex or empty.

Then, there exists $x_{0} \in A$ such that $\left(x_{0}, y\right) \notin M$ for all $y$ $\in A$.

Lemma $2.3^{10}$ Let $A$ be a non-empty compact convex subset of a locally convex Hausdorff vector topological space X. If $F: A \rightrightarrows A$ is upper semi-continuous and for any $x \in A, F(x)$ is non-empty, convex and closed, then there exists $x^{*} \in A$ such that $x^{*} \in F\left(x^{*}\right)$. 


\section{MAIN RESULTS}

In this section, we establish some existence theorems of solution sets for (SQVEP).

Definition 3.1 Let $X, Y, Z$ be topological vector spaces and $C \subset Z$ be a closed convex point cone with int $C$ $\neq \varnothing$, where int $C$ denotes the interior of $C$. Suppose $h: X, Y, X \longrightarrow Z$ be a vector function. $\mathrm{h}$ is said to be strongly $\mathrm{C}$-quasiconvex (with respect to the first variable) in a set $A \subset X$, if for each $y \in Y, z \in X$ and $\forall x_{1}, x_{2}$ $\in A, \forall \lambda \in[0,1], h\left(x_{1}, y, z\right) \in C$ and $h\left(x_{2}, y, z\right) \in C$. Then, it follows that

$$
h\left(\lambda x_{1}+(1-\lambda) x_{2}, y, z\right) \in C .
$$

Theorem 3.1 Suppose that for the problem (SQVEP) that

i) $K$ and $T$ are continuous in $A \times B$ with non-empty compact convex values;

ii) for all $(x, y) \in A \times B, f(x, y, x) \in C$ and $g(x, y, x) \in C$

iii) the set $\left\{\left(y, x^{*}\right) \in B \times A\right.$ : $\left.f\left(., y, x^{*}\right) \notin C\right\}$ is convex in $A$ and the set

$\left\{\left(x, y^{*}\right) \in A \times B: g\left(., x, y^{*}\right) \notin C\right\}$ is convex in $B$;

iv) for all $\left(y, x^{*}\right) \in B \times A, f\left(., y, x^{*}\right)$ is strongly $C$ quasiconvex in $A$, and for all $\left(x, y^{*}\right) \in A \times B, g\left(., x, y^{*}\right)$ is strongly $C$-quasi-convex in $B$;

v) the set

$\left\{\left(x, y, x^{*}\right) \in A \times B \times A: f\left(x, y, x^{*}\right) \in C\right\}$ is closed, and the set $\left\{\left(y, x, y^{*}\right) \in B \times A \times B: g\left(y, x, y^{*}\right) \in C\right\}$ is closed.

Then, the (SQVEP) has a solution, i.e., there exists $(\bar{x}, \bar{y})$

$\in A \times B$ such that $\bar{x} \in S(\bar{x}, \bar{y}), \bar{y} \in T(\bar{x}, \bar{y})$ and

$$
\begin{aligned}
& f\left(\bar{x}, \bar{y}, x^{*}\right) \in C, \forall x^{*} \in K(\bar{x}, \bar{y}), \\
& g\left(\bar{y}, \bar{x}, y^{*}\right) \in C, \forall y^{*} \in T(\bar{x}, \bar{y}),
\end{aligned}
$$

Moreover, the solution set of the (SQVEP) is closed.

Proof. For all $(x, y) \in A \times B$, define mappings: $\Pi_{1}: A \times$

$B \rightrightarrows A$ and $\Pi_{2}: A \times B \rightrightarrows B$ by

$$
\Pi_{1}(x, y)=\left\{\begin{array}{c}
a \in K(x, y): f\left(a, y, x^{*}\right) \in C, \\
\forall x^{*} \in K(x, y)
\end{array}\right\},
$$

and

$$
\Pi_{2}(x, y)=\left\{\begin{array}{c}
b \in T(x, y): g\left(b, y, x^{*}\right) \in C, \\
\forall y^{*} \in T(x, y)
\end{array}\right\},
$$

Firstly, we will show that $\Pi_{1}(x, y)$ and $\Pi_{2}(x, y)$ are non-empty.

Indeed, for all $(x, y) \in A \times B, K(x, y)$ is non-empty compact convex set. Setting

$$
M=\left\{\begin{array}{l}
\left(a, x^{*}\right) \in K(x, y) \times K(x, y): \\
f\left(a, y, x^{*}\right) \notin 0
\end{array}\right\}
$$

(1) The condition (ii) we have, for any

$$
a \in K(x, y),(a, a) \notin M
$$

(2) The condition (iii) implies that for any $a \in K(x, y)$, $\left\{x^{*} \in K(x, y):\left(a, x^{*}\right) \in M\right\}$ is convex in $K(x, y)$.

3) The condition (v), we have for any $a \in K(x, y)$, $\left\{x^{*} \in K(x, y):\left(a, x^{*}\right) \in M\right\}$ is open in $K(x, y)$.

By Lemma 2.2, there exists $a \in K(x, y)$ such that $\left(a, x^{*}\right)$ $\notin M$ for all $x^{*} \in K(x, y)$, i.e.,

$$
f\left(a, y, x^{*}\right) \in C, \forall x^{*} \in K(x, y) .
$$

Thus $\Pi_{1}(x, y)=\varnothing$. Similarly, we also have $\Pi_{2}(x, y)=$ $\varnothing$.

Secondly, we will prove that $\Pi_{1}(x, y)$ and $\Pi_{2}(x, y)$ are non-empty convex sets.

Let $a_{1}, a_{2} \in \Pi_{1}(x, y)$ and $\alpha \in[0,1]$ and let $a=$ $\alpha a_{1}+(1-\alpha) a_{2}$. Since $a_{1}, a_{2} \in K(x, y)$ and $K(x, y)$ is a convex set, we have $a \in K(x, y)$. Thus, for $a_{1}, a_{2} \in$ $\Pi_{1}(x, y)$, it follows that

$$
f\left(a_{1}, y, x^{*}\right) \in C, \forall x^{*} \in K(x, y),
$$

and

$$
f\left(a_{2}, y, x^{*}\right) \in C, \forall x^{*} \in K(x, y) .
$$

By (iv), $f\left(., y, x^{*}\right)$ is strongly $C$-quasi-convex.

$$
f\left(t a_{1}+(1-t) a_{2}, y, x^{*}\right) \in C, \text { for all } t \in[0,1],
$$

i.e. $a \in \Pi_{1}(x, y)$. Therefore, $\Pi_{1}(x, y)$ is convex. Similarly, we have $\Pi_{2}(x, y)$ is convex.

Thirdly, we will proof that $\Pi_{1}$ and $\Pi_{2}$ are upper semicontinuous in $A \times B$ with non-empty compact values. Firstly, we will show that $\Pi_{1}$ is upper semi-continuous in $A \times B$ with nonempty compact values. Indeed, since $A$ is a compact set, by Lemma 2.1(ii), then we will show that $\Pi_{1}$ is a closed mapping. Indeed, let a net

$$
\left\{\left(x_{\alpha}, y_{\alpha}\right): \alpha \in I\right\} \subset A \times B
$$

such that

$$
\left(x_{\alpha}, y_{\alpha}\right) \rightarrow(x, y) \in A \times B
$$

and let $a_{\alpha} \in \Pi_{1}\left(x_{\alpha}, y_{\alpha}\right)$ such that $a_{\alpha} \longrightarrow a_{0}$. Now we need to show that $a_{0} \in \Pi_{1}(x, y)$. Since $a_{\alpha} \in$ $K\left(x_{\alpha}, y_{\alpha}\right)$ and $K$ is upper semi-continuous with non-empty compact values, hence $\mathrm{K}$ is closed, thus, we have $a_{0} \in K(x, y)$. Suppose the contrary $a_{0} \notin$ $\Pi_{1}(x, y)$. Then, there exists $x_{0}^{*} \in K(x, y)$ such that

$$
f\left(a_{0}, y, x_{0}^{*}\right) \notin C .
$$

By the lower semi-continuity of $K$, there is a net $\left\{x_{\alpha}^{*}\right\}$ such that $x_{\alpha}^{*} \in K\left(x_{\alpha}, y_{\alpha}\right), x_{\alpha}^{*} \rightarrow x_{0}^{*}$. Since $a_{\alpha} \in$ $\Pi_{1}\left(x_{\alpha}, y_{\alpha}\right)$, we have

$$
f\left(a_{\alpha}, y_{\alpha}, x_{\alpha}^{*}\right) \in C
$$


By the condition (v) and (3.2), we have

$$
f\left(a_{0}, y, x_{0}^{*}\right) \in C \text {. }
$$

This is the contradiction between (3.1) and (3.3). Thus $a_{0} \in \Pi_{1}(x, y)$. Hence, $\Pi_{1}$ is upper semi-continuous in $A \times B$ with nonempty compact values. Similarly, we also have $\Pi_{2}$ is upper semi-continuous in $A \times B$ with non-empty compact values.

Next, we need to prove the solutions set $\Omega \neq \varnothing$.

Define the set-valued mappings

$\Phi_{1}: A \times B \rightrightarrows A \times A$ and $\Phi_{2}: A \times B \rightrightarrows B \times B$ by

$$
\Phi_{1}(x, y)=\left(\Pi_{1}(x, y), K(x, y)\right), \forall(x, y) \in A, B
$$

and

$$
\Phi_{2}(x, y)=\left(\Pi_{2}(x, y), K(x, y)\right), \forall(x, y) \in A, B
$$

Then $\Phi_{1}, \Phi_{2}$ are upper semi-continuous and $\forall(x, y) \in$ $A \times B, \Phi_{1}(x, y)$ and $\Phi_{2}(x, y)$ are non-empty compact convex subsets of $A \times B$.

Define the set-valued mapping

$$
H:(A \times B) \times(A \times B) \rightrightarrows(A \times A) \times(B \times B)
$$

by

$$
H((x, y),(x, y))=\left(\Phi_{1}(x, y), \Phi_{2}(x, y)\right)
$$

Then $H$ is also upper semi-continuous and $\forall(x, y) \in$ $A \times B, H((x, y),(x, y))$ is a nonempty closed convex subset of $(A \times B) \times(A \times B)$.

By Lemma 2.3 , there exists a point $((\widehat{x}, \widehat{y}),(\widehat{x}, \widehat{y})) \in$ $(A \times B) \times(A \times B)$

such that

$$
((\widehat{x}, \widehat{y}),(\widehat{x}, \widehat{y})) \in H((\widehat{x}, \widehat{y}),(\widehat{x}, \widehat{y}))
$$

that is

$$
((\widehat{x}, \widehat{y}),(\widehat{x}, \widehat{y})) \in \Phi_{1}(\widehat{x}, \widehat{y}),(\widehat{x}, \widehat{y}) \in \Phi_{2}(\widehat{x}, \widehat{y})
$$

which implies that $\widehat{x} \in \Pi_{1}(\widehat{x}, \widehat{y}), \widehat{y} \in K(\widehat{x}, \widehat{y})$ and $\widehat{x} \in$ $\Pi_{2}(\widehat{x}, \widehat{y}), \widehat{y} \in T(\widehat{x}, \widehat{y})$. Hence,

$$
\widehat{x} \in K(\widehat{x}, \widehat{y}), \widehat{y} \in T(\widehat{x}, \widehat{y})
$$

and

$$
f\left(\widehat{x}, \widehat{y}, x^{*}\right) \in C, \forall x^{*} \in K(\widehat{x}, \widehat{y}),
$$

and

$$
g\left(\widehat{y}, \widehat{x}, y^{*}\right) \in C, \forall y^{*} \in T(\widehat{x}, \widehat{y}),
$$

i.e., (SQVEP) has a solution.

Finally, we prove that $\Omega$ is closed. Indeed, let a net $\left\{\left(x_{\alpha}, y_{\alpha}\right), \alpha \in I\right\} \subset \Omega:\left(x_{\alpha}, y_{\alpha}\right) \longrightarrow\left(x_{0}, y_{0}\right)$. We need to prove that $\left(x_{0}, y_{0}\right) \in \Omega$. Indeed, by the lower semi-continuity of $\mathrm{K}$ and $\mathrm{T}$, for any

$$
x_{0} \in K\left(x_{0}, y_{0}\right), y_{0} \in T\left(x_{0}, y_{0}\right) \text {, }
$$

there exist $x_{\alpha} \in K\left(x_{\alpha}, y_{\alpha}\right), y_{\alpha} \in T\left(x_{\alpha}, y_{\alpha}\right)$ such that $x_{\alpha} \longrightarrow x_{0}, y_{\alpha} \longrightarrow y_{0}$. Since $\left(x_{\alpha}, y_{\alpha}\right) \in \Omega$, we have $x_{\alpha} \in K\left(x_{\alpha}, y_{\alpha}\right), y_{\alpha} \in T\left(x_{\alpha}, y_{\alpha}\right)$ such that

$$
f\left(x_{\alpha}, y_{\alpha}, x_{\alpha}^{*}\right) \in C, \forall x_{\alpha}^{*} \in K\left(x_{\alpha}, y_{\alpha}\right),
$$

and

$$
g\left(y_{\alpha}, x_{\alpha}, y_{\alpha}^{*}\right) \in C, \forall y_{\alpha}^{*} \in T\left(x_{\alpha}, y_{\alpha}\right) .
$$

Since $K, T$ are upper semi-continuous in $A \times B$ with nonempty compact values. There exist $x_{0}^{*} \in K\left(x_{0}, y_{0}\right)$ and $y_{0}^{*} \in T\left(x_{0}, y_{0}\right)$ such that $x_{\alpha}^{*} \longrightarrow x_{0}^{*}, y_{\alpha}^{*} \longrightarrow y_{0}^{*}$ (taking subnets if necessary). By the condition (v) and

$$
\left(x_{\alpha}, y_{\alpha}, x_{\alpha}^{*}, y_{\alpha}^{*}\right) \rightarrow\left(x_{0}, y_{0}, x_{0}^{*}, y_{0}^{*}\right),
$$

we have

$$
f\left(x_{0}, y_{0}, x_{0}^{*}\right) \in C, \forall x_{0}^{*} \in K\left(x_{0}, y_{0}\right),
$$

and

$$
g\left(y_{0}, x_{0}, y_{0}^{*}\right) \in C, \forall y_{0}^{*} \in T\left(x_{0}, y_{0}\right) .
$$

This means that $\left(x_{0}, y_{0}\right) \in \Omega$. Thus $\Omega$ is a closed set. Remark 3.2 If $K(x, y)=K(x), T(x, y)=$ $T(x), \quad g\left(y, x, y^{*}\right)=f\left(x, y, x^{*}\right)$ with $x \in A$, $y \in A, x^{*} \in A, y^{*} \in B$. Then, (SQVEP) will be the strong vector quasi-equilibrium problem which was studied in ${ }^{11}$. Hou et al. ${ }^{11}$ also obtained an existence result for strong vector quasi-equilibrium problem. However, the assumptions and proof methods of Theorem 3.1 in $^{11}$ are new and different from that in Theorem 3.1.

By the following example, we show that in the special case as Remark 3.2, all the assumptions of Theorem 3.1 are satisfied. But, Theorem 3.1 in $^{11}$ we cannot apply these conditions. It is because $f$ is not $(-C)$ continuous.

Example 3.3 Let $X=Y=Z=\mathrm{R}, A=B=[0,2], C=$ $\mathrm{R}_{+}$

and let

$$
K: A \times B \rightrightarrows A, T: A \times B \rightrightarrows B
$$

and

$$
f: A \times B \times A \rightarrow Z, g: B \times A \times B \rightarrow Z
$$

be defined by

$$
K(x, y)=T(x, y)=[0,2],
$$




$$
\begin{aligned}
& f\left(x, y, x_{0}\right)=g\left(y, x, y_{0}\right) \\
& =\left\{\begin{array}{l}
{[0,2], \text { if } x_{0}=y_{0}=\frac{3}{2},} \\
{[3,4] \text { otherwise }}
\end{array}\right.
\end{aligned}
$$

We show that all assumptions of Theorem 3.1 are satisfied. However, $f$ is not $(-C)$-continuous at $x_{0}=y_{0}=$ $\frac{3}{2}$.

Thus, it gives case where Theorem 3.1 can be applied but Theorem 3.1 in $^{11}$ does not work.

Remark 3.4 If we let

$$
\begin{aligned}
& f\left(x, y, x^{*}\right)=f\left(x^{*}, y\right)-f(x, y), \\
& g\left(y, x, y^{*}\right)=g\left(x, y^{*}\right)-g(x, y)
\end{aligned}
$$

with $x \in A, y \in A, x^{*} \in A, y^{*} \in B$ and replace $C$ by $\mathrm{Z}$-int C. Then, (SQVEP) will be changed to symmetric vector quasi-equilibrium problem which is studied in $^{2}$. In $^{2} \mathrm{Fu}$ obtained an existence result for symmetric vector quasi-equilibrium problem. However, the assumptions and proof methods of Theorem in ${ }^{2}$ are also new and different from that in Theorem 3.1.

In the following example, we show that in the special case as Remark 3.4, all the assumptions of Theorem 3.1 are satisfied. But, Theorem 3.1 in $^{11}$ and Theorem in $^{2}$ can not be applied.

Example 3.5 Let $X=Y=Z=\mathrm{R}, A=B=[0,3], C=$ $\mathrm{R}_{+}$

and let

$$
K: A \times B \rightrightarrows A, T: A \times B \rightrightarrows B
$$

and

$$
f: A \times B \times A \rightarrow Z, g: B \times A \times B \rightarrow Z
$$

be defined by

$$
\begin{gathered}
K(x, y)=T(x, y)=[0,3], \\
f\left(x, y, x_{0}\right)=g\left(y, x, y_{0}\right) \\
=\left\{\begin{array}{l}
{[0,2], \text { if } x_{0}=y_{0}=\frac{1}{4},} \\
{\left[0, \frac{1}{3}\right] \text { otherwise }}
\end{array}\right.
\end{gathered}
$$

We show that all assumptions of Theorem 3.1 are satisfied. However, $f$ is neither $C$-continuous nor properly C-quasi-convex at $x_{0}=y_{0}=1 / 4$. Therefore, Theorem 3.1 can be applied but Theorem 3.1 in $^{11}$ and Theorem in $^{2}$ do not work.

Theorem 3.6 Suppose that for the problem (SQVEP) assumptions (i), (ii), (iii) and (iv) as in Theorem 3.1 and the condition (v) can be replaced by the following condition:

(v') f and $g$ are continuous.

Then, the (SQVEP) has a solution, i.e., there exists $(\bar{x}, \bar{y})$ $\in A \times B$ such that

$$
\bar{x} \in K(\bar{x}, \bar{y}), \bar{y} \in T(\bar{x}, \bar{y})
$$

and

$$
\begin{aligned}
& f\left(\bar{x}, \bar{y}, x^{*}\right) \in C, \forall x^{*} \in K(\bar{x}, \bar{y}), \\
& g\left(\bar{y}, \bar{x}, x^{*}\right) \in C, \forall y^{*} \in T(\bar{x}, \bar{y}) .
\end{aligned}
$$

Moreover, the solution set of the (SQVEP) is closed.

Proof. We omit the proof since the technique is similar as that for Theorem 3.1 with suitable modifications.

\section{CONCLUSION}

The results presented in this paper improve and extend the main results in the literature. Some examples are given to illustrate our results. Hence our results, Theorem 3.1 and Theorem 3.6 have significant improvements.

\section{ACKNOWLEDGMENTS}

This research is funded by Ho Chi Minh City University of Technology-VNU-HCM under grant number T-KHUD-2018-84.

\section{CONFLICT OF INTEREST}

We declare that there is no conflict of whatsoever involved in publishing this research.

\section{AUTHOR CONTRIBUTIONS}

All authors contributed equally to this work. All authors have read and agreed to the published version of the manuscript.

\section{REFERENCES}

1. Noor MA, Oettli W. On general nonlinear complementarity problems and quasi-equilibria. Mathmatiche XLIX;1994:313331.

2. Fu JY. Symmetric vector quasi-equilibrium problems. J. Math. Anal. Appl. 2003;285:708-713. Available from: https://doi.org/ 10.1016/S0022-247X(03)00479-7.

3. Chen B, Huang NJ, Cho YJ. Symmetric strong vector quasiequilibrium problems in Hausdorff locally convex spaces. J. Inequal. Appl. 2011;56:1-15. Available from: https://doi.org/ 10.1186/1029-242X-2011-56.

4. Fakhar M, Zafarani J. Generalized symmetric vector quasiequilibrium problems. J. Optim. Theory Appl. 2008;136:397-409. Available from: https://doi.org/10.1007/s10957-007-9310-1.

5. Lin $\mathrm{Z}$, Yang $\mathrm{H}, \mathrm{Yu}$ J. On existence and essential components of the solution set for the system of vector quasi-equilibrium problems. Nonlinear Anal. 2005;63:2445-2452. Available from: https://doi.org/10.1016/j.na.2005.03.049.

6. Aubin JP, Ekeland I. Applied Nonlinear Analysis. John Wiley and Sons, New York. 1984;

7. Luc DT. Theory of Vector Optimization: Lecture Notes in Economics and Mathematical Systems. Springer-Verlag Berlin Heidelberg. 1989;Available from: https://doi.org/10.1007/9783-642-50280-4.

8. Ferro F. A minimax theorem for vector-valued functions. J. Optim. Theory Appl. 1989;60:19-31. Available from: https: //doi.org/10.1007/BF00938796.

9. Fan K. A generalization of Tychonoff's fixed point theorem. Math Ann. 1961;142:305-310. Available from: https://doi.org/ 10.1007/BF01353421.

10. Holmes RB. Geometric Functional Analysis and its Application. Springer-Verlag, New York. 1975;Available from: https: //doi.org/10.1007/978-1-4684-9369-6.

11. Hou SH, Gong XH, Yang XM. Existence and stability of solutions for generalized Ky Fan inequality problems with trifunctions. J. Optim. Theory Appl. 2010;146:387-398. Available from: https://doi.org/10.1007/s10957-010-9656-7. 